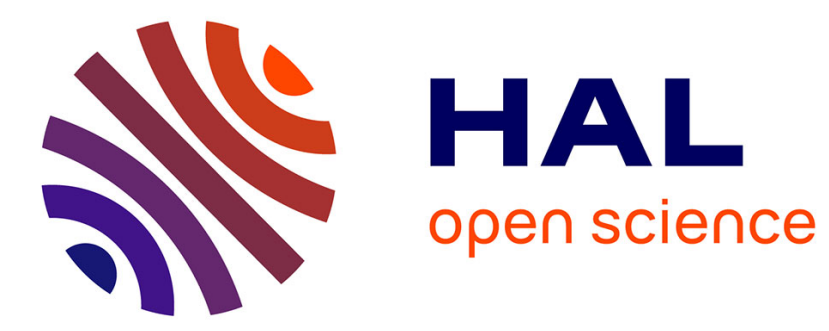

\title{
Bodily Reminiscences
}

Houria Abdelouahed

\section{To cite this version:}

Houria Abdelouahed. Bodily Reminiscences. Topique - Revue freudienne, 2009, L'acte poétique, 4 (109), pp.9 - 29. 10.3917/top.109.0022 . hal-01508595

\section{HAL Id: hal-01508595 \\ https://hal.science/hal-01508595}

Submitted on 19 Jun 2017

HAL is a multi-disciplinary open access archive for the deposit and dissemination of scientific research documents, whether they are published or not. The documents may come from teaching and research institutions in France or abroad, or from public or private research centers.
L'archive ouverte pluridisciplinaire HAL, est destinée au dépôt et à la diffusion de documents scientifiques de niveau recherche, publiés ou non, émanant des établissements d'enseignement et de recherche français ou étrangers, des laboratoires publics ou privés. 


\title{
Adonis ou la réminiscence du corps
}

\author{
Houria Abdelouahed
}

«Riche en mérites, c'est poétiquement pourtant que l'homme habite sur cette terre»

HöLDERLIN

\section{RÉMINISCENCE DU CORPS}

«Le corps se souvient », écrit Adonis ${ }^{1}$.

«Où sont-elles passées les hystériques de jadis, ces femmes merveilleuses, les Anna O, les Dora?...», disait Lacan.

Devant l'extension du champ de la clinique et l'intérêt aujourd'hui pour les pathologies narcissiques et les états limites, est-ce un appel à cette figure matricielle de la psychanalyse qui a bien su tisser un lien indissoluble entre la psychanalyse et le corps? Entre la sexualité et la psychanalyse ? Est-ce parce que l'hystérique n'est plus un objet d'attention comme elle le fut pour la psychanalyse naissante? Appel nostalgique, alors, à la matrice théorique qui donna naissance à la psychanalyse après avoir focalisé pendant des siècles l'intérêt

1. Adonis, Commencement du corps fin de l'océan, Mercure de France, 2004. Adonis est un poète contemporain né en 1930 en Syrie. Il est l'auteur d'une œuvre importante : Le fixe et le mouvant (en 4 volumes), Mémoire du vent, Chants de Mihyar le damascène, Al-Kitâb (Le Livre en 3 volumes)... Ami des grands poètes français Alain Bosquet, André du Bouchet, Alain Jouffroy...il a traduit en arabe Baudelaire, Michaux et Saint John Perse. Adonis a révolutionné la poésie arabe en rompant aussi bien avec le thème qu'avec la forme classique. Sa poésie prône la rupture également avec la religion et avec la philosophie platonicienne.

Topique, 2009, 109, 9-27. 
autour de l'animal utérin rebelle, instable, voyageur ou migrateur, et ce depuis le Papyrus Kahun, le plus ancien des textes médicaux connus de l'Antiquité, jusqu'à Charcot pour lequel fut créée la première chaire mondiale de clinique des maladies nerveuses. Et si l'intérêt de Charcot fut, dit-on, de libérerl'hystérie de l'étiologie utérine traditionnelle, il n'en demeura pas moins, comme l'attestent ses fameuses Leçons du mardi, que l'hypersthésie ovarienne resta à l'honneur devant le public médusé de la Salpêtrière et que l'anamnèse se résumait au rappel du quotidien difficile de la classe ouvrière, nécessitant ainsi les conseils du maître ( «faites-la moins travailler ${ }^{2} »$, dit-il à la mère de la malade). Les leçons du mardi offraient des descriptions minutieuses des états du corps rappelant l'approche des anatomistes dans leur désir d'en transcender le mystère. Si pour l'anatomie, le corps ne cache rien qui ne puisse être rendu visible par le recours à la dissection, note Pierre Fédida, pour Charcot, le corps ne cache rien à un visuel qui sait prononcer le discours du maître ${ }^{3}$. L'éthique du voir dominait, en effet, le tableau, prenant ainsi le devant de la scène, occultant toute narrativité. Devant le regard des «messieurs » de la Salpêtrière, les gesticulations théâtrales de l'hystérique répondaient à la voix du maître. Torsion, abasie, paralysies, anesthésie, aphonie, respiration coupée ou accélérée, évanouissement, névralgie, troubles fonctionnels... Tout est là excepté, comme le note J.-B. Pontalis, l'espace psychique qui sera le grand absent. Il a fallu tout un chemin pour que Freud rompe avec le voir au profit de l'écoute de ce qui se trame dans l'Autre scène. Désormais, on est prié de fermer les yeux et ... d'écouter.

Écouter quoi ? D'abord l'interdit de toucher. L'hystérique qui se voua corps et âme au maître afin de sauver la forme, dira à Freud: «Ne me touchez pas ! Ne dites rien ! \& L'interdit du toucher et le silence imposé à l'analyste sont nécessaires pour pouvoir entendre que torsion, paralysies, anesthésie, aphonie, respiration coupée ou accélérée, évanouissement, névralgie, troubles fonctionnels... sont des substituts de la satisfaction sexuelle. L'endroit douloureux de la cuisse est celui qui recevait, jadis, le contact de la jambe du père aimé. L'hystérique a su ouvrir un écart entre voir et entendre, entre regarder et écouter. Les possibilités figuratives habitent, désormais, le récit et deviennent de l'ordre de l'entendu. Ainsi, «l'idéal de l'exhaustif descriptif » s'efface au profit d'une oreille qui voit la plasticité d'un corps parlé. « à notre surprise, dit Freud, nous découvrîmes, en effet, que chacun des symptômes hystériques disparaissait immédiatement et sans retour quant on réussissait à mettre en pleine lumière le souvenir de l'incident déclenchant et à éveiller l'affect lié à ce dernier et quant ensuite, le malade décrivait ce qui lui était arrivé (...) en donnant à son émotion une expression verbale ${ }^{4}$. » 114.

2. Charcot J.-M., «Leçon du mardi » (1887-88), in L'hystérie, L'Harmattan, 1971, pp. 111-

3. Fédida P., « L'anatomie dans la psychanalyse », in N.R.P., $\mathrm{n}^{\circ}$ 3, 1971, pp. 109-126.

4. Freud S \& Breuer J., Etudes sur l'hystérie, PUF, 1981, p. 4 
« Ce que tu cherches, cela est proche et vient déjà à ta rencontre », dit Hölderlin. Dans cet intervalle, qui verra le surgissement d'une parole instituant l'interdit du toucher au profit de la «juste distance des corps », Freud, dans son attention flottante, est proche du poète qui reçoit le surgissement de la parole poétique. L'interdit proféré par l'hystérique : «ne dites rien » trouva une résonance chez celui qui attendait ce qui était déjà en train de cheminer vers lui et allait à sa rencontre. La distance et le silence imposés par l'hystérique sont reçus par celui qui ne saisit que trop bien que la parole, qui est un substitut à l'acte, est le seul garant de l'existence d'un espace psychique. Néanmoins, il ressemble à celui qui revient au pays natal dans le poème d'Hölderlin. L'arrivant doit encore chercher. «Ce qu'il cherche vient déjà à sa rencontre; cela est proche. Mais ce qu'il cherche n'est pas encore trouvé », écrit Heidegger au sujet de l'arrivant au pays natal ${ }^{5}$. L'hystérique restera encore, enveloppée dans son mystère, « difficile à gagner, la Renfermée » (Hölderlin).

Dans la notice nécrologique qu'il consacre à Charcot, Freud insiste sur la dimension visuelle chez ce dernier: «Ce n'était pas quelqu'un qui rumine, ni un penseur, mais une nature artistiquement douée (...) un visuel, un voyant ${ }^{6}$. ». Or, toute la distance est là. Grand lecteur des classiques (Sophocle, Shakespeare, Goethe, Schiller...), Freud, qui écrit sur l'aphasie, le pouvoir magique du mot, ne pouvait qu'être réceptif à l'injonction de l'hystérique. «Ne dites rien ! signifie: «écoutez».

«Depuis que nous sommes un dialogue

Et que nous pouvons ouïr les uns les autres » (Hölderlin).

L'hystérique (ou Freud) rompt avec la captation visuelle au profit de ce qui traverse siècles et générations, depuis l'Antiquité jusqu'à nos jours, à savoir le motif de la parole.

Or, loin d'être une simple conséquence du fait de parler les uns avec les autres, le pouvoir entendre en est bien au contraire la présupposition ${ }^{7}$. Entendre se trouve érigé sur la possibilité de la parole. Les deux co-existent. Et ce, depuis l'origine. «Il fut le premier locuteur et je fus le premier auditeur. Ainsi, le monde est né grâce à la parole », dit Ibn Arabi qui a su donner toute son importance à la prédisposition de celui qui reçoit la parole.

C'est dans l'article où Freud évoque cette nature visuelle de Charcot, qu'il s'interroge : «d'où vient-il que l'hystérique est soumis à un affect dont il affirme ne rien savoir du motif ${ }^{8}$ ? » En fait, que se trame-t-il dans l'arrière scène ? Que tait-elle en fait? Quelle est cette chose qui ne parvient pas à s'articuler ? De quoi le corps est-il le gardien ? «L'analyste fait remonter la conversion d'un

5. Heidegger, Approche de Hölderlin, Gallimard, coll Tel, 1973, p. 16.

6. Freud S., «Charcot» (1893), in Résultats, idées, problèmes, I., PUF, 1984, p. 62.

7. Heidegger, op. cit., p. 49.

8. Freud S., op. cit., p. 69. 
émoi psychique en douleur physique à une époque où cette douleur ne fut certainement pas ressentie et ne fit pas l'objet d'un souvenir'». Car l'hystérique souffre de réminiscence. Toutefois, puisque «les mots qui surgissent savent de nous ce que nous ignorons d'eux » (René Char), elle dira que l'endroit originellement douloureux à la cuisse droite avait un lien avec les soins donnés au père.

Ainsi, cette phrase de Charcot: « une douleur vive, très vive même ; les malades ne peuvent supporter le moindre attouchement» se trouve mise en mal par l'attitude d'Elisabeth qui montre une satisfaction si l'on pinçait la peau ou les muscles hyperalgiques, ou si l'on exerçait une pression sur eux. Ses traits prenaient une singulière expression de satisfaction plutôt que de douleur, note Freud. L'hystérique fonctionnait comme si l'anatomie n'existait pas, ou comme si elle n'en avait nulle connaissance. Celui qui écoute, quant à lui, ne parle pas d' «excitation corticale», mais de «déguisement » et de «représentation».

Le corps dont parle l'hystérique est le corps érogène en lien avec le désir inconscient et la dimension fantasmatique. Là, nous découvrons que l'hystérique ignore les lois de l'anatomie. La sienne est fantastique car fantasmatique. Et le corps qui est pris dans les rets du signifiant est un corps tissé de mouvements pulsionnels. Aussi renvoie-t-il à l'érotique et ses impasses. Du corps physiologique naît le corps érotique de par une subversion libidinale des fonctions physiologiques et l'émergence de la sexualité. Aussi la psychologie à l'usage des neurologues laisse-t-elle à l'arrière plan le corps biologique ou le corps des anatomo-physiologistes au profit d'un corps construit, défini dans et par son érogénéité et son fonctionnement aussi bien fantastique que fantasmatique en lien avec la préhistoire du sujet. «Les représentations émanant des plus grandes profondeurs et qui constituent le noyau même de l'organisation pathogène sont celles auxquelles les malades ont le plus de peine à attribuer le caractère de souvenirs ${ }^{10}$ » ou bien «les phénomènes moteurs de l'accès hystérique peuvent être considérés, en partie, comme des formes réactionnelles ordinaires de l'affect lié au souvenir (...) en partie comme des mouvements exprimant un souvenir ${ }^{11}$.»

Saisissant au vol l'interdit du toucher afin qu'une parole puisse dire le corps érogène, écrivant que la chose qui tiraille et ne cesse de se dire à sa manière n'est autre que la chose sexuelle, Freud ne rompt pas seulement avec l'enseignement du maître delaSalpêtrière, maiségalementavecl'idéalisme platonicien qui met l'âme au devant de la scène condamnant le corps à en être le simple tombeau.

9. Freud S., Etudes sur l'hystérie, p. 117.

10. Etudes sur l'hystérie, p. 146.

11. Ibid., p. 243 et p. 11 . 


\section{LE CORPS COMME MÉTHODE}

Même le discours de Diotime qui a séduit des générations de philosophes, ne fait que renvoyer vers un au-delà du corps. «La vie pour un homme vaut la peine d'être vécue, quand il contemple le beau en lui-même», dit l'étrangère de Mantinée ${ }^{12}$.

Or, «quel sens ont ces notions auxiliaires de la morale : «âme», «esprit», libre arbitre », «Dieu», si ce n'est celui de ruiner physiologiquement l'humanité. Lorsqu'on détourne le sérieux de la volonté de survivre et d'accroître ses forces physiques, c'est-à-dire lorsqu'on le détourne de la vie, lorsqu'on fait de l'anémie un idéal, du mépris du corps un chimérique «salut de l'âme», qu'estce donc sinon la recette même de la décadence ? », écrit Nietzsche ${ }^{13} s^{\prime}$ insurgeant contre l'idéalisme ascétique qui conduit à cette dépréciation ontologique du corps. «Sans le fil conducteur du corps je ne crois à la validité d'aucune recherche». Contrairement à l'âme qui, dans la tradition platonicienne, a la capacité d'atteindre l'intelligible, le corps est condamné parce qu'il est menacé de décrépitude, de corruption, de transformation et d'instabilité, et comme tel, il ne peut être que du côté du leurre. Or, du moment où les sens nous montrent « le devenir, l'écoulement, le changement, rétorque Nietzsche, ils ne mentent pas ${ }^{14} \gg$. Ainsi, « je suis un corps de part en part et rien hors cela ».

La question du souvenir dans la tradition platonicienne débouche sur une aporie: «Et comment t'y prendras-tu, Socrate, pour chercher une chose dont tu ne connais pas du tout ce qu'elle est ? " Et ce dernier de répondre : «Vois-tu bien quelles disputes soulève ce sujet que tu mets sur le tapis, qu'il n'est pas possible à l'homme de chercher ni ce qu'il sait, ni ce qu'il ne sait pas ? Il ne saurait chercher ce qu'il sait, puisqu'il le sait, et qu'en ce cas, il n'a pas besoin de le chercher, ni ce qu'il ne sait pas pour la raison qu'il ne sait même pas ce qu'il doit chercher. » Laquelle aporie tente à se résoudre par le recours à l'âme. «Puisque l'âme est immortelle, dit Socrate, et qu'elle a vécu plusieurs vies, et qu'elle a vu tout ce qui se passe, ici et dans l'Hadès, il n'est rien qu'elle n'ait appris. Aussi n'est-il pas du tout surprenant que, sur la vertu et sur le reste, elle puisse se souvenir de ce qu'elle a su auparavant. Comme tout se tient dans la nature et que l'âme a tout appris, rien n'empêche qu'en se rappelant une seule chose, et que les hommes appellent apprendre, elle ne retrouve d'elle-même toutes les autres, pourvu qu'elle soit courageuse et ne se lasse pas de chercher; car chercher et apprendre n'est autre chose que se ressouvenir ${ }^{15}$. »

12. Dans le discours de Diotime, il faut commencer par reconnaître la beauté visible et sensible qui se trouve dans le corps, puis les dépasser pour découvrir la beauté de l'âme vertueuse et finalement s'élever encore pour atteindre la beauté transcendante qui est le principe de toutes les beautés. (Platon, Le Banquet, Les Belles lettres, 211d).

13. Nietzsche F., Ecce Homo, cité par Wolting P., in Le corps, (sous la direction de J.-C. Goddard), Vrin, 2005, p. 176

14. Nietzsche F., Crépuscule des idoles, p. 173.

15. Platon, Le Menon, Flammarion, 1967, p. 343. 
Si apprendre, c'est inviter l'enfoui à refaire surface, il n'en demeure pas moins que la nature du souvenir et la nature de l'oublié sont entièrement différentes dans l'optique platonicienne et dans celle de Freud. Sensible à l'enseignement de Nietzsche ${ }^{16}$, Freud, de la même façon qu'il arrache le corps de l'hystérique au discours médical, rompt avec la vision philosophique classique. Le souvenir ne suppose plus la vue immédiate et directe des Idées (que nous avions lorsque nous vivions avec les dieux), mais devient le savoir de l'Autre scène. Et si l'hystérique souffre de réminiscence, son corps se charge de le lui rappeler. Le corps se souvient de la trace de plaisir éprouvée. Lequel plaisir est nécessairement sexuel. Offrant le dispositif clinique qui permettra que le corps se dégage du discours philosophique et physiologique pour le définir dans son érogénéité et sonéconomie fantasmatique, l'hystérique invite à dégager la question de la réminiscence de l'optique platonicienne. L'hystérique cherche ce qu'elle sait déjà. Elle sait, mais d'un savoir inconscient. Il faut la juste distance des corps et l'interdit du toucher afin que les mots nomment, dans une levée du refoulement, la chose sexuelle. Le corps demeure le gardien de ce qu'elle sait. La réminiscence implique, désormais, le pouvoir du corps de dire la chose sexuelle. « Le procédé thérapeutique supprime l'action de la représentation primitive non abréagie en permettant la liquidation, par expression verbale, de l'affect concomitant». Anna O. baptisera ce procédé du nom de «talking-cure ». Elle parlera également de «Shimney sweeping».

\section{« ANGÉLIQUE ANIMALITÉ »}

$\mathrm{Si}$ «c'est de réminiscence que souffre surtout l'hystérique» (Freud), «le corps se souvient» (Adonis). Ainsi, il cesse d'être un simple «entrelacs de vision et de mouvement» (M. Merleau-Ponty), pour devenir le corps érogène, celui qui exprime le «cheminement du désir dans la géographie de la matière (Adonis). Et ce, dans une rencontre avec l'autre.

«Si tu possédais l'être aimé, écrit Bataille, ce cœur que la solitude étrangle formerait un seul cœur avec celui de l'être aimé17 ${ }^{17}$.

«De toi je m'habille»

avec toi je m'unifie ${ }^{18} »$.

Toutefois, dans l'élan amoureux et la confusion des deux êtres, la discontinuité. Dans le désir de se rejoindre, règnent la mort, la béance, l'abîme.

«Toujours, il y eut entre nous une béance». Et dans l'abolition des distances :

16. « Nietzsche où je trouverai, je l'espère, des mots pour beaucoup de choses qui restent muettes en moi », écrit Freud. Cf. Assoun P.-L., Freud et Nietzsche, Quadrige/PUF, 1998.

17 Bataille G., L'érotisme, Les éditions de Minuit, 1957, p. 27.

18 Adonis, Singuliers, in Chants de Mihyar le Damascène, Poésie/Gallimard, 2002. 
«Un pont nous conjoint que nous ne pouvons franchir

Un rempart nous unit / nous sépare ${ }^{19}$. »

Le mouvement de l'amour, porté à l'extrême, est un mouvement de mort (Bataille). « Ma mort est une échelle d'accès à mon corps ${ }^{20}{ }$.

Le poète dit que la sexualité humaine n'est point rudimentaire, que l'érotisme est le propre de l'humain. Seul ce dernier est porté sur la transgression, l'excès, le fantasme:

«Que de nourrices, j’ai détournées de leurs nourrissons aux amulettes.

Derrière elle si ses pleurs fusaient, elle lui tendait le sein

Tandis que sous moi le bas de son corps s'offrait ${ }^{21}$. »

Condensation des deux images, celle de la mère et l'autre de l'amante qui en dit long sur la dimension fantasmatique au cœur de la sexualité humaine.

Dans ce cheminement du désir, le corps devient langage(Nietzsche) ou alphabet (Adonis). Mais non pas «un alphabet du visible du corps », comme l'écrit Georges Didi-Huberman au sujet de $\mathrm{Charcot}^{22}$, ce dernier ne s'étant guère soucié de déchiffrer ce qui se présente comme au-delà de ce qui se donne à voir, mais un alphabet qui dit l'inscription dans la géographie de la matière, voire plusieurs au plus profond de la chair ou «la voûte charnelle» (Supervielle).

Et

«Un homme, un homme, une femme» (Adonis).

Si le poète dit dans un premier temps la continuité des êtres à partir de leur discontinuité, que Jean-Pierre Vernant résume magnifiquement par : $1+1=3$, ici il résume de façon inouïe le dédoublement. Lorsque j'ai demandé à Adonis d'expliquer le vers poétique, il a avoué que ce n'était guère aisé à un poète de reprendre en prose, ce moment de surgissement.

Dans Blessures de mémoires ${ }^{23}$, Michel Schneider retraçant l'histoire du mouvement psychanalytique, nous invite à saisir ce qu'il en est de ce dédoublement et des identifications mutuelles à la lumière de la relation Freud/Fliess. Relation qui passe par le corps d'une femme. On connaît l'histoire. Fliess oublie la bande de $50 \mathrm{~cm}$ de gaze. La malade eut une hémorragie et perdit connaissance. Emma manqua de mourir. Cinquante centimètres de gaze comme l'équivalent d'un pénis dans le corps de la femme. «Message phallique ». Le sang d'Emma, s'interroge Michel Schneider, comme relève du sperme de Fliess ? On en décèle la trace devant l'horreur de Freud devant « ces larges escarres blanc grisâtre ». Freud rêve de l'injection faite à Irma. Le corps d'Emma donnera le rêve princeps. Emma est le pont qui permet à Freud de passer de Charcot à Breuer, de 1'hyp-

19. Adonis, Singuliers.

20. Op. cit.

21. Imru'u al-Qays (poète préislamique), cf. Abdelouahed H. \& Adonis, Dîwân de la poésie arabe classique, Poésie/Gallimard, 2008.

22. Didi-Huberman G., L'invention de l'hystérie, Macula, 1982.

23. Schneider M., Blessures de mémoire, Gallimard, 1980. 
notisme à l'interprétation du rêve. Ce rêve d'Irma est la matrice théorique d'où est sortie la psychanalyse. Ainsi, l'opération d'Emma est la scène primitive de la psychanalyse. Scène qui «image la rencontre d'un homme et d'une femme dans la conjonction d'un homme et de son autre soi-même» (Michel Schneider).

«Un homme, un homme, une femme ».

Est-ce l'autre spéculaire? Dans ce jeu des identifications mutuelles et des soins réciproques, se dit néanmoins le recours à une différence, le corps de la femme. «Au-delà de l'autre, l'Autre entre les deux», dira Michel Schneider ${ }^{24}$.

«Une femme, une femme, un homme» (Adonis).

Faceàl'homme, undédoublement desfiguresféminines.Emma-Irma/Freud. Le féminin redouble-t-il la maternité? Un sang que la femme a perdu, qui ne fera jamais retour, «y compris en l'engrossant d'une interprétation, elle qui a porté dans son corps, l'enfant pansement que Fliess a fait à Freud: la psychanalyse ${ }^{25} \gg$. Redoublement également de la figure féminine, la femme et la mère, la mère derrière lafemme.

Le poète, double de l'analyste ${ }^{26}$ ?

\section{LA FINE SENSIBILITÉ}

«Nous autres profanes, écrit Freud, nous avons toujours été curieux de savoir où cette singulière personnalité, le créateur littéraire, va prendre sa matière (...) et comment il parvient, par elle, à tellement nous saisir, à provoquer en nous des émotions dont nous ne serions peut-être même pas crus capables ${ }^{27}$ ». Si on les interrogeait, continuait Freud, ils seraient incapables de nous fournir une réponse satisfaisante. Freud dira plus tard: «le fait que les poètes ont des qualités leur permettant de venir à bout d'une telle tâche : avant tout une fine sensibilité qui leur fait percevoir les mouvements cachés de l'âme d'autrui, et le courage de laisser parler leur propre inconscient ${ }^{28}{ } \gg$.

J. Mc Dougall, se proposant de réfléchir sur l'artiste et l'analyste, se contente d'examiner «des symptômes etinhibitions susceptibles d'engendrerdes conflits dans le monde interne des $\operatorname{artistes}^{29} \gg$. Dans la même mouvance, Michel de M'Uzan, de l'interrogation sur l'essence de la sublimation artistique qui échappe

24. Schneider M., op.cit., p. 41

25. Op. cit., p.45-46

26. C'est la question que pose Monique Schneider dans son article «Freud et le combat avec l'artiste », in L'artiste et le psychanalyste, PUF, Petite bibliothèque de psychanalyse, 2008, p. 48.

27. Freud, «Le créateur littéraire et la fantaisie», in L'inquiétante étrangeté et autres essais, Gallimard, coll. Essais, 1985, p. 33.

28. Freud, «Un type particulier de choix d'objet chez l'homme », in La vie sexuelle, PUF, p. 47.

29. Mc Dougall J., «L'artiste et le psychanalyste », in L'artiste et le psychanalyste, op. cit., p. 13. 
«à notre discipline », en vient à évoquer et analyser les cas des patients confrontés aux affres de la création ${ }^{30}$. Or, si Freud nous demande de nous adresser au poète, c'est parce que ce dernier a cette capacité de dire, comme en une étincelle, cette vérité inconsciente à laquelle l'analyste arrive après un travail laborieux. Le poète moderne disant «Que l'amour est profond dans sa haine !» (Adonis) rejoint Shakespeare faisant dire à Juliette : «Je t'aime du fond de ma haine». La haine se trouve ainsi à l'origine de l'élan amoureux, ou bien «Seul celui qui est amoureux voit sa propre image dans l'eau ", dit Rûmî, dans un dépassement de la mélancolie de Narcisse et dans une ouverture sur l'écart et l'espace de la représentation. «Tu vois un reflet dans le miroir, est-ce voir véritablement ton visage ?»(Attâr). Seules les expériences extrêmes montent «le visage réel, cru » (S. Le Poulichet) ou encore « Oublieuse mémoire », disait Claudel tant il est vrai que l'effacement est nécessaire à l'épreuve d'écriture.

La parole poétique transcende temps et espace. André du Bouchet écrivant : $\mathrm{Si}$

la hauteur de l'air

a

suffi

la parole

le

donne

dépassée alors

ou

en

fleur (Dans leurs voix, les eaux)

rejoint superbement à dix siècles d'intervalle, de l'Orient à l'Occident, cette phrase de Omar Khayâm : «Je dis l'alef; mon cœur reprit : 'n'en dis pas davantage'». Ibn Arabi dira : «Sans la verticalité de l'air, le monde n'aurait pas existé ${ }^{31}$ ».

Réfléchir sur la création artistique à partir des affres de la névrose, fait penser à cette remarque de Winnicott : «Quand la psychanalyse a tenté de s'attaquer au problème de la créativité, elle $\mathrm{a}$, pour une grande part, perdu de vue le thème principal ; les auteurs ont préféré porté leur choix, dans le domaine de la créativité artistique, sur une personnalité hors du commun et hasarder des observations d'ordre secondaire ou tertiaire ; mais ce que l'on pourrait appeler le primaire, ils l'ont ignoré. Certes, on peut prendre, par exemple, Léonard de Vinci comme objet d'études et faire des commentaires pleins d'intérêt sur la relation entre l'œuvre de l'artiste et certains événements de sa petite enfance (...) Mais mettre au jour des relations de ce genre, quand on se penche sur des hommes et des

30. De M’Uzan M., « Aperçus sur le processus de la création littéraire », in De l'art à la mort, Gallimard, col Tel, 1977.

31. Pour les poètes de la mystique musulmane, cf. Abdelouahed H., La visualité du langage, L'Harmattan, 1998, «La source et l'écart», in Cliniques méditerranéennes, ${ }^{\circ}$ 73, 2006. 
femmes célèbres, éloigne du thème qui est au centre de l'idée de créativité (...) La direction de l'investigation est mauvaise au départ. Le thème principal se dérobe $^{32}{ }^{\gg}$.

Freud, qui refusait de faire de Nietzsche un cas de pathographie, écrit : «Les poètes et les romanciers sont de précieux alliés, et leur témoignage doit être estimé très haut, car ils connaissent entre ciel et terre, bien des choses que notre sagesse scolaire ne saurait encore rêver ${ }^{33}$.»

Joyce Mc Dougall, évoquant la violence chezles créateurs, recourt au modèle kleinien $^{34}$. Or, outre le fait que le plaisir esthétique semble omis, il y a lieu de distinguer, me semble-t-il, entre le fait d'exposer sa création, ce qui suppose un rapport à un public, et la violence de l'acte créatif en lui-même en lien avec cette tension qu'on peut rapprocher de ce que Freud décrit dans « Pour introduire le narcissisme» : «On se met à aimer pour ne pas tomber malade». En grand philologue, Ibn Arabi se sert de la notion de karab (tension, tristesse) pour expliquer cet état de souffrance, de douleur et de tension d'avant la création. Al-karab revêt une signification économique puisqu'il dicte la nécessité d'une décharge. Décharge qu'Attâr exprime de cette façon : «Si je ne parle pas, je brûle». Plus proche de nous, Francis Ponge parle de l'orage originel qui «en nous a grondé».

\section{LA MATIÈRE POÉTIQUE}

Il est frappant de constater que les auteurs qui se penchent sur le travail de l'artiste négligent de rappeler qu'il existe une différence entre les artistes quant à leur matière.

Ce qui caractérise l'acte poétique c'est qu'il est taillé dans l'expérience langagière ou la matière du langage. Ce dernier est ce qui sert à la communication. Seulement, son essence ne se résume pas dans le fait qu'il soit l'instrument de communication. Le système de mots et de règles syntaxiques n'est que l'aspect extérieur du langage. Walter Benjamin définit l'essence du langage par sa capacité de nommer les choses. «Dans l'acte de nomination demeure l'essence spirituelle du langage », écrit-il ${ }^{35}$. Cette essence, Heidegger la définit comme ouverture de l'étant. À ce moment, la poésie qui est fondation par la parole l'est également dans la parole. Et si elle est «l'occupation la plus innocente»

32. Winnicott, « La créativité et ses origines », in Jeu et réalité, Gallimard, p. 97.

33. Freud S., Délire et rêve dans la Gradiva de Jensen, Gallimard, col. Idées, 1949, p. 127.

34. «En dehors de la force et de l'intensité du processus créateur lui-même, les artistes sont des êtres violents en ce qu'ils cherchent à exercer leur pouvoir sur le monde extérieur, c'est-àdire à imposer leurs pensées, leurs images, leurs rêves ou leurs cauchemars. » Op.cit, p.12-13

35. «Dans le nom l'essence spirituelle qui se communique est le langage », écrit Walter Benjamin, Mythe et violence, Denoë1, 1971, p. 83. 
(Hölderlin) car elle se manifeste sous la forme la plus discrète du jeu, il n'en demeure pas moins qu'elle s'exprime dans « le plus dangereux des biens » (Hölderlin), à savoir le langage. Par le langage, peuvent s'exprimer «ce qu'il y a de plus pur et de plus abscons, aussi bien que le confus et le commun ${ }^{36} \gg$. La poésie ne reçoit pas le langage comme une matière à œuvrer et qui serait à sa disposition, mais c'est au contraire la poésie qui commence par rendre possible le langage, dit Heidegger ${ }^{37}$. J'ajoute que le langage, le lieu de la métaphore, du transport amoureux et la sensorialité des mots à la manière de l'enfant qui joue («La terre est bleue comme une orange», Paul Eluard) et qui en dit long sur la sexualité infantile, est également celui de l'aridité ou du désossement effrayants comme en témoigne la psychose.

Creusant dans sa «terre verbeuse », le poète taille à la manière du sculpteur de per via di levare. Dans la masse opaque de la matière, ici langagière, qu'en «fera mon ciseau ? Sera-t-il Dieu, table ou cuvette ${ }^{38}$ ? », s'interroge-t-il. On peut dire, à l'instar de Lacan, qu'on coupe avec des signifiants, sans toutefois oublier que dans «la rage de l'expression», il y a «le parti pris des choses » (Francis Ponge).

Puisant dans la matière verbale, et laissant surgir à son insu dans un instant de dessaisissement une parole, tranchante, décisive, incisive, le psychanalyste est proche du poète. L'œuvre d'Adonis qui dit magnifiquement « la pulsion d'exhumer» (Sophie de Mijolla) en lien avec un véritable travail de construction et d'imagination (Face au trauma, le poète, à l'instar de l'analyste, n'a plus que sa capacité imaginative ${ }^{39}$ ), dit également l'inquiétante étrangeté de la demeure première. Poète et analyste sont du côté de ce que Freud nomme «la croyance animiste». «Dans la psychanalyse n'est-il pas requis de l'analyste que son recours animiste soit précisément cette primitivité qui le rend immédiatement sensible à l'inquiétante étrangeté de l'autre et donc de soi-même ${ }^{40}$ ? »

Toutefois, au-delà de l'angoisse que provoque le natal, au-delà de l'inquiétante étrangeté de la demeure familière, le poète nous met face à l'unheimlich au sein de la langue, une inquiétante étrangeté propre au langage ${ }^{41}$. Dans notre réflexion sur la poésie, nous aurons plus à gagner en pensant au primitif qu'au jeu des pulsions archaïques et prégénitales.

Et lorsque Freud écrit que «quelque chose vient rabaisser la valeur de ce qu'ils (les poètes) nous communiquent; ainsi ne peuvent-ils représenter la

36. Ibid., p. 47.

37. Heidegger M., op. cit., p. 55

38. Marin L., « Trois gloses», N.R.P., $\mathrm{n}^{\circ} 44,1991$, p. 129

39. Pierre Fédida a su donner toute son importance à cette question de la puissance imaginative chez l'analyste. Cf. Humain, déshumain, PUF, Petite bibliothèque de psychanalyse, 2007.

40. Petitier Aline, «Une inquiétude en marche», in L'inactuel, Nouvelle série, $\mathrm{n}^{\circ} 2,1999$, p. 188.

41. Gomez-Mango E., «Le pays natal», in La mort enfant, Gallimard, 2003. 
réalité telle quelle sans l'avoir modifiée ${ }^{42}{ }^{2}$, on peut rétorquer que loin de rabaisser le poète, cette modification est indispensable à l'acte poétique. Nécessaire, sinon on serait dans une pensée opératoire qui se contente de rapporter la réalité sans aucune teinte fantasmatique. «Il en est d'autres, ne l'oublions pas, qui sont si solidement ancrés dans la réalité objectivement perçue qu'ils sont malades, mais dans la direction opposée : ils ont perdu le contact avec le monde subjectif et se montrent incapables de toute approche créative de la réalité ${ }^{43}{ }^{\prime}$. Le poète ne photographie pas le monde. Il dit la subjectivité, explore la mémoire sensorielle, se délecte des mots, de leur pouvoir magique, tente d'approcher la chose qui... fuit. D'où le jeu avec les mots. Jeu qui dit le sexuel infantile.

La psychanalyse a vu le jour grâce à l'hystérie, une œuvre d'art déformée. Lorsque cessa le jeu éblouissant du scopique, ou l'excès de visibilité, cette oeuvre d'art a pu exprimer le sexuel et le désir inconscient. Lorsque l'analyste se tait, l'organe est dans la parole. Le corps parlé est le corps vécu, éprouvé, habité, il est celui de la subjectivité. «Tout authentique penser, et tout authentique penser en compagnie d'un penseur, est voyage - qui se met en route pour atteindre ce qui est déjà tout proche et saute aux yeux et qui n'est autre que le tout simple. C'est seulement dans un tel voyage qu'il y a expérience », dit Heidegger ${ }^{44}$. Cette parole de Heidegger résume l'expérience freudienne qui a su reconsidérer le corps.

Le langage est aux origines de la psychanalyse car il est lié au corps libidinal. Il faut penser le corps à partir de ce qui en nous est «pensant», « voulant», «sentant», disait Nietzsche, et «s'éprouvant», ajoutent aussi bien le psychanalyste que le poète.

Houria ABDELOUAHED 255, rue d'Épinay 95360 Montmagny houriya_abdelouahed@yahoo.fr

42. Freud S., La vie sexuelle, p. 47.

43. Winnicott D.W., op. cit., p. 93.

44. Heidegger M., op.cit., p. 121. 


\section{Houria Abdelouahed - Réminiscence du corps}

Résumé : Parlant d'un souvenir du corps, l'analyste, à l'instar du poète, rompt avec l'ascétisme platonicien (qui définit le corps comme un simple tombeau de l'âme) et institue le corps comme un lieu du savoir. La réminiscence devient pouvoir du corps de dire et d'exprimer la chose sexuelle. Le corps, qui est pris dans les rets des signifiants, est traversé par le désir inconscient et les mouvements pulsionnels. En outre, taillant dans la matière langagière, l'analyste laisse surgir à son insu dans un instant de dessaisissement une parole tranchante, incisive. À ce moment, il devient proche du poète. L'un et l'autre, explorent la croyance animiste et l'inquiétante étrangeté du langage.

Mots-clés : Le corps - Le sexuel - La matière verbale - L'inquiétante étrangeté Déssaisissement - Croyance animiste.

\section{Houria Abdelouahed -Bodily Reminiscences}

Summary : When an analyst addresses the question of a memory inscribed in the body, then, just like the poet, he or she breaks away from Platonic asceticism (which posits the body as a simple tomb in which the soul resides) and establishes the body as a place instilled with knowledge. Such reminiscences are ways for the body to voice and elaborate on things of an inherently sexual nature. The body, caught up in the nets of signification, is permeated by unconscious desire and drives. Moreover, in cutting into the matter of language itself, the analyst may unwillingly, in a moment of relinquishment, utter an incisive and insightful word. At this moment, he or she is almost as much of a poet than an analyst, for both explore animist beliefs and the uncanny nature of language.

Key-words : The Body - The Sexual - Verbal Matter - The Uncanny Relinquishment - Animist Belief. 


\section{Entretien avec Adonis}

Puisant dans sa terre verbeuse, Adonis écrit : «Le corps se souvient».

La voix du poète rejoint la pensée psychanalytique définissant le corps comme tissé de mouvements pulsionnels et d'incidents érotiques, corps portant la marque du plaisir.

À l'encontre, donc, de l'idéalisme ascétique du Phédon - où le corps est la prison de l'âme - et d'une certaine tradition philosophique où le corps est défini comme un objet doué d'extension, donné dans une représentation et accessible à une connaissance consciente -, s'élève une exigence de pensée que partagent aussi bien le poète que l'analyste, exigence qui institue le corps comme le lieu où se déploie la subjectivité.

Et au sein du corpus arabe moderne, Adonis, dans sa rupture avec la pensée théologique et dogmatique, et ce depuis Les Chants de Mihyar le damascène jusqu'à Histoire qui se déchire sur le corps d'une femme ${ }^{2}$, opère une révolution poétique et intellectuelle. À l'instar de Nietzsche, il fait du corps une méthode. Mais, fervent lecteur d'Héraclite et de la pensée mystique, il casse la pensée de l'Un (de la théologie) au profit du corps qui devient l'un-multiple.

Cet entretien permet de mesurer l'ampleur d'une telle révolution.

\section{ENTRETIEN AVEC ADONIS ${ }^{1}$}

\section{LE CORPS : UN MULTIPLE}

Houria - Deux termes, dans la langue arabe, désignent le corps : al-jism et aljasad.

Adonis - N'oublie pas al-badan.

Houria - Il existe en effet ce troisième terme. L'Encyclopédie d'Ibn Manzûr², stipule une différence entre jism qui désigne le corps de l'animal et jasad qui est le corps humain. Ibn Manzûr écrit : «il est ce qui s'alimente». Cela paraît curieux carl'animal s'alimenteégalement, saufà dire que «s'alimenter» dépasse le cadre de la nourriture au sens classique.

1. Extraits in Adonis avec Houria Abdelouahed, Le regard d'Orphée, Fayard, 2009.

2. Adonis, Chants de Mihyar le damascene, traduit par Anne Wade Minkowski, 1961, Actes Sud ; Gallimard, col. Poésie, 2002.

Adonis, Histoire qui se déchire sur le corps d'une femme, trad. Houria Abdelouahed, Mercure de France, 2008. 
Adonis - En effet, car al-jasad a une dimension érotique. Nous ne parlons jamais du jasad lorsque nous évoquons les anges par exemple.

Houria - C'est le mot «jasad» que tu utilises dans Commencement du corps fin de l'océan pour parler du corps. Tout ton recueil traite, en effet, de la dimension érotique du corps. À te lire, ce dernier n'est pas simplement un entrelacs de vision et de mouvement (selon l'expression de Merleau-Ponty), mais le lieu érotique par excellence. En t'éloignant de la philosophie, tu rejoins la psychanalyse qui institue le début de la sexualité humaine dans la rencontre : bouche-mamelon. L'introduction de la sexualité dans le monde de l'enfantcommence par la bouche qui découvre, à travers l'alimentation, le plaisir érogène.

Adonis - Premièrement, je ne sais si on peut traduire jasad par corps. Jasad est plus intériorisé, plus érotique. En revanche, jism est plus général. Deuxièmement, le discours sur al-jasad doit être pris dans le cadre d'une culture religieuse islamique qui annule toute réflexion sur le corps. Alors que sur le plan de la pratique individuelle, elle permet à l'homme de jouir, sans limite, du corps, tout en réduisant la femme à n'être qu'un instrument ou un lieu pour la réception de la substance masculine. Ma réflexion sur le commencement du corps est en contradiction avec la religion qui stipule un commencement dans l’âme. Ma poésie, mon écriture et ma vie disent le contraire.

Houria - Je n'ai pas lu ton recueil dans le sens d'un corps qui serait premier, mais dans le sens suivant: le commencement du corps est la fin de l'océan, à savoir que ce corps est dépourvu d'un commencement. Me revient, alors, la question de Bataille : «Par où commence le corps humain ?» Bataille écrit : «La bouche est le commencement ou si l'on veut la proue des animaux dans le cas le plus caractéristique. Elle est la partie la plus vivante, c'est-à-dire la plus terrifiante pour les voisins. Mais l'homme n'a pas une architecture simple comme les bêtes. Il n'est même pas possible de dire où il commence. »

Adonis - Il y deux aspects, celui dont tu parles, puis un autre qui a un lien, comme je l'ai dit, avec le contexte religieux. Dire que le corps n'a pas de commencement, revient à dire qu'il est un océan.

Houria - Avant de revenir sur l'infini du corps, j'ai envie de dire que le terme de sharj (anus, tu parles précisément, dans Commencement du corps fin de l'océan, d'un lieu de jouissance entre le sexe et l'anus) est totalement absent de la poésie arabe moderne, alors que la littérature ancienne regorge de passages sur l'importance érotique de l'anus. Éjaculer dans le sharj, lit-on chez Nafzaoui, est le summum de ce que l'homme peut atteindre comme jouissance. J'ai lu dans l'un de ces ouvrages anciens que certaines mères conseillaient à leurs filles de se donner librement à leurs compagnons, de dire, lorsque ces derniers arrivent à la jouissance, des mots obscènes en offrant leur sharj.

Adonis - Tu peux dire que le terme est, aujourd'hui, inexistant.

Houria - Freud écrit : « Dommage que je ne puisse pas m'embrasser moimême», Adonis écrit : «Comme si je désirais mon corps ». 
Adonis - Mais je n'ai commencé à lire Freud que depuis notre rencontre, et seulement par rapport à des questions qui portent sur la religion.

Houria - Pierre Fédida, au sujet de cette nostalgie de complétude sexuelle, écrit que la bouche est « ouverte sur une parole qui n'en finit pas mais qui, surtout, ne procède d'aucun commencement ». Pour Pierre Fédida, l'ouverture sur la bouche de la parole est une ouverture sur l'origine du corps.

Adonis - Mais quelle est l'origine du corps? Le corps n'a pas d'origine, car il a toutes les origines.

Il y a un point dans l'activité sexuelle qui est l'orgasme, point où se mêle la vie et la mort, ne serait-ce qu'un instant et que chacun de nous éprouve. D'un côté, l'acte sexuel est le sommet de la saisie humaine et de l'autre, c'est un effacement de cette saisie. Toutefois, c'est un effacement pour un nouveau commencement. L'acte sexuel est un plus dans la conscience de soi ou la saisie de soi. Et s'il y a une absence, c'est celle des personnes qui arrivent à plonger dans les profondeurs du fleuve afin d'en sortir avec plus de vie. La mort dans la sexualité est une sorte de vie. Et la rencontre sexuelle suppose une connaissance. Le corps doit connaître l'autre corps afin de vivre la rencontre sexuelle. Si je ne connais pas le corps de la femme, mon corps ne pourrait pas rencontrer le sien. Il y a des choses très simples mais extrêmement importantes et qui changent la nature de l'acte sexuel.

Houria - Le corps n'est-il pas l'infini de la parole humaine sur le corps luimême?

Adonis - Le problème du corps, n'est pas du côté du tangible et du sensible, mais ce qui est à l'arrière plan. Le corps est formé par plusieurs étages : un étage sensible, un autre influencé par celui-ci et un troisième en rapport avec l'esprit et la réflexion. En fait, je pense que le corps n'a pas eu l'étude qu'il méritait, même en Occident. Peut-être que Freud a donné des clefs essentielles pour réfléchir sur le corps, mais ces clefs n'ont pas été suffisamment explorées. C'est un sujet bikr (vierge), comme le corps. Vierge car infini. Et le plaisir est toujours nouveau. C'est un perpétuel mouvement de création et de recréation. Le corps se crée lui-même. Mais il ne se crée que lorsqu'il est avec un autre corps. Et pour moi, le corps est celui de la femme. Abû Tammâm dit : «L'amour est pour le premier aimé ». Car c'est le premier contrat entre l'amoureux et le monde. Et il s'agit là de l'amour dans son acception sexuelle. Car il renvoie à la première fois où s'effectue l'amour entre l'être et le monde, ou entre l'amoureux et son amant. Le corps est important pour le poète, mais il n'a pas eu l'intérêt qu'il méritait. Le corps est un continent incroyable. Il n'y a pas un sexe qui ressemble à un autre sexe, un sein qui ressemble à un autre sein, une taille semblable à une autre, ni une bouche à une autre. Le corps ne se répète pas. Il n'y a pas de répétition dans le monde du corps. C'est un continent détruit par la religion. Pour sortir de cette destruction et découvrir le corps, il faut tout un travail.

Houria - Je trouve que c'est un point extrêmement fort dans ta poésie et il 
me semble que tu as fait un travail singulier et pionnier. Tu dé-théologises la religion au profit du corps. C'est à travers le corps que se rencontrent, chez toi, athéisme et mystique. L'aversion nietzschéenne pour la religion rencontre la pensée d'Ibn Arabi lorsqu'il dit: «Dieu ne se manifeste pas deux fois. »C'est ce que tu écris et exprimes au sujet du corps. Le triangle des mystiques (le connaissant, le connu et la connaissance) devient dans ta poésie le triangle du corps dans l'acte amoureux et sexuel.

Adonis - Le plus important de ce que j'ai écrit, figure dans Singuliers. Il y a un corps comme il y a une spiritualité. Mais, s'il y a une spiritualité c'est celle du corps. Il n'y a pas d'âme. Il y a un continent qui s'appelle le corps. Mais un seul corps suffit-il pour connaître le corps? Je parle aussi bien de l'homme que de la femme. Ce sont les lois sociales, les coutumes et les traditions qui les lient à un seul corps.

Houria - Lorsque tu rencontres une femme, explores-tu le corps pour explorer la langue ou ce n'est que plus tard que tu penses à l'alphabet des membres ?

Adonis - Non, je n'exploite pas et je n'instrumentalise pas. Lorsqu'on dit que le corps est infini, il ne s'agit pas seulement de mon corps ou de ton corps, mais bel et bien du corps comme concept. Il devient infini dans ce sens là. Je me découvre à la lumière d'un corps et j'exprime ce que je découvre dans cette rencontre avec ce corps.

Houria - La satisfaction du désir n'est jamais totale. C'est dans la nature du désir que de se tenir comme insatisfait. Néanmoins, à rabattre cette insatisfaction sur la réalité qui est la multiplicité des corps, fait courir le risque de tomber dans «la morale du névrosé », à savoir ce n'est jamais celui-là, c'est toujours l'autre. Celui qui est ailleurs.

Adonis - Je parle d'un sens profond et positif, comme l'être humain qui ne peut se découvrir qu'à travers plusieurs étapes. Aujourd'hui, tout en habitant le même corps, je suis très différent de ce que j'étais il y a 20 ans ou 50 ans. Alors que je suis toujours moi-même, je n'ai pas encore découvert mon moi. Dans ce sens, l'autre corps aide à se découvrir, bien qu'on soit dans le mouvement de l'infini.

Houria - Le corps n'est-il pas à chaque fois différent ?

Adonis - C'est un monde immense. Il se peut que les religions aient inventé le mariage pour limiter cet infini. Si l'homme aime l'inconnu, le corps de la femme est un grand inconnu. Dans cette rencontre avec le corps de la femme, on découvre l'inconnu du monde. Et entrer dans le corps de la femme, c'est entrer dans cet inconnu du monde.

Houria - Le sexe de la femme se dit mahbal en arabe (le lieu de l'angoisse et de la folie). Or, la littérature arabe moderne, de façon générale, ne s'arrête jamais sur cette angoisse. Ne serait-ce que lors de la première rencontre. Et comme nous l'avons déjà souligné, le mot $a$ 'dhar (qui désignerait vierge pour le masculin) n'existe pas.

3. Encyclopédie philologique du XIII siècle. 
Adonis - Je n'aime pas parler de moi-même. Mais je pense être le seul qui ait tenté de réfléchir sur le corps, en particulier dans Singuliers. Le corps, chez les Arabes, relève plutôt de la poésie courtoise. Il reste une belle architecture pour embellir la vie et la rendre plus joyeuse, surtout si la femme devient mère. Le corps, à ce moment-là, va de pair avec la transformation de la femme en un élément du décor.

Houria - Dans l'échange entre Adam et ève, tel qu'il est relaté par Tabarî, la femme est désignée comme le lieu de l'apaisement, et non de l'angoisse. Or, l'angoisse etle féminin sontindissolublement liés. D'oùl'intérêt pourles Arabes, aujourd'hui, de lire leur corpus à la lumière de la bouche d'Irma ${ }^{4}$.

Adonis - Tout-à-fait. Le monde du corps est extrêmement riche, complexe et difficile. Il y a des parties du corps et des instants dont il est difficile de parler. Des instants qui se vivent comme on respire, comme on étreint la lumière du jour, comme on boit. Une partie organique de la vie. Le corps est deux. Il n'y a pas de corps un. Il est deux lorsque nous parlons de l'amour.

Houria - «Il y a deux affirmations de l'amour, écrit R. Barthes. Tout d'abord lorsque l'amoureux rencontre l'autre, il y a affirmation immédiate (...) je dis oui à tout (en m'aveuglant). Suit un long tunnel: mon premier oui est rongé de doutes. La valeur amoureuse est sans cesse menacée de dépréciation : c'est le moment de la passion triste (...) De ce tunnel cependant, je puis sortir, je puis « surmonter », sans liquider; ce que j'ai affirmé une première fois, je puis de nouveau l'affirmer, sans le répéter. Car alors, ce que j'affirme, c'est l'affirmation, non sa contingence : $\mathrm{j}$ 'affirme la première rencontre dans sa différence, je veux son retour non sa répétition. Je dis à l'autre (ancien ou nouveau) : Recommençons. » Le lecteur d'Héraclite et de Nietzsche que tu es, se sent-il proche de ce discours de R. Barthes malgré toutes ces années qui ont passé et malgré les cheveux blanchis par le temps?

Adonis - Je me sens très proche de ce qui est soulevé ici par R. Barthes. Mais on aime dans un corps, ce qu'on n'aime pas dans un autre corps. Je pense que tout ce qui a été écrit sur le corps, ne peut s'écrire que selonl'expérience de chacun. L'importance du corps et sa grandeur, c'est qu'il échappe à la généralisation. Le corps dépasse toutes les généralités.

Houria - Barthes parle, justement à la fin, du non-vouloir saisir. Il conclut par: «verra jamais.»

Adonis- C'est l'énigme et le secret du corps. Le corps est un continent qui n'a pas été exploré. Et ce continent est toujours disposé à être découvert de façon

4. De la racine h.b.l., qui signifie la folie.

5 Le rêve de l'injonction faite à Irma, figure dans L'interprétation des rêves de Freud. Lacan qui, lie la bouche à l'angoisse et au féminin, écrit : «Il y a là une horrible découverte. Celle de la chair qu'on ne voit jamais, le fond des choses, l'envers de la face (...) La chair dans tout son sort, au plus profond du mystère. » et il poursuit: «Vision d'angoisse, identification d'angoisse », Le Séminaire, Livre II. Le moi dans la théorie de Freud et dans la technique psychanalytique, Seuil, 1978, p. 186. 
continue. Raison pour laquelle le corps reste plus important que l'esprit. Il est plus ouvert que l'esprit et n'a aucune logique. L'esprit et la logique ont des critères qui les limitent. Tandis que le corps est une puissance extraordinaire. Cette puissance peut se trouver chez l'individu qui est capable de découvrir le corps de façon continue.

Houria - Mais singulière.

Adonis - à chaque fois que j'évoque le corps, je me souviens d'Héraclite disant : «tu ne traverses pas le fleuve deux fois ». Tu ne rencontres pas deux fois le même corps. Il est comme l'atome que tu ne peux toucher. Tu ne peux le toucher car il est déjà ailleurs. Le corps est une forêt d'atomes.

Houria - Le «je t'aime», dit encore Barthes, laisse flotter une sorte de: on ne peut rien dire de l'amour. L'amour reste un défi pour l'expérience langagière. Que l'on parle de ravissement, de foudre ou d'énamoration, quelque chose échappe à la parole.

Adonis - Absolument. On ne peut s'exprimer sur l'amour. Seul l'amour exprime l'amour. L'exprimer, c'est le limiter et le réduire. Ce qu'il y a de plus beau dans le corps, c'est qu'il résume superbement le monde. Il contient ce qu'il y a de plus vil et ce qu'il y a de plus élevé dans le monde.

Houria - Le mérite de l'expérience sexuelle, c'est qu'elle réveille l'attrait de ce qui a été comprimé par la civilisation car jugé comme anal ou vil.

Adonis - Le corps est un résumé de ce qui constitue le commencement du monde et sa fin. Il y a la bouche, en haut, et l'anus en bas. Les deux sont au même niveau et pourtant, différents l'un de l'autre. Pas de corps sans la bouche et sans l'anus.

Houria - Tu rejoins Bataille.

Adonis - Bataille insiste davantage sur l'anus. Il ne considère pas un ensemble poétique et homogène avec une partie qui lie l'une à l'autre, une partie qui serait la sœur del'autre.

Houria - L'anus n'est pas le contraire de la bouche. C'est ce que tu es en train de dire?

A- Il n'est pas l'antinomie. Chacun complète l'autre.

$\mathrm{H}-\mathrm{Je}$ pense que c'est Deleuze qui ne comprend pas que la bouche puisse baiser, car elle est le lieu de l'immondice.

Adonis - Ce qui est le plus beau dans la relation sexuelle, c'est quand la bouche de l'amant palpe le sexe de l'amante. Le sexe de la femme est une autre bouche pour la bouche de l'amant.

Houria - P. Fédida a raison de dire que le corps ne se construit qu'à travers la parole de la bouche. Il a raison, à condition de ne pas spiritualiser le corps. Il faut donner toute sa portée sexuelle et pleinement sensorielle à cette expérience de la bouche qui dit le corps. J'ai l'impression qu'on l'oublie par moment. Tu nous invites, une fois de plus, à rompre avec la pensée platonicienne. 